\title{
Potential impacts of the Montreal Protocol Kigali Amendment to the choice of refrigerant alternatives
}

\author{
Fabio Polonara $^{1,5^{*}}$, Lambert J.M. Kuijpers ${ }^{2,5}$, Roberto A. Peixoto ${ }^{3,4,5}$ \\ ${ }^{1}$ Università Politecnica delle Marche, Ancona, Italy \\ ${ }^{2}$ A/genT Environmental Consultancy, Venlo, The Netherlands \\ ${ }^{3}$ Istituto Mauà de Tecnologia, Sao Paulo, Brazil \\ ${ }^{4}$ London South Bank University, London, UK \\ ${ }^{5}$ UNEP, Refrigeration Technical Options Committee, RTOC
}

Email: f.polonara@univpm.it

\begin{abstract}
The paper first gives a description of the "Kigali Amendment" established HFC phase-down as decided by the Montreal Protocol parties in Kigali, Rwanda, October 2016. Baselines, freeze dates, schedules for the reductions of HFC consumption for both developing and developed country groups, as well as the 17 HFCs that are now included in the Montreal Protocol are summarized. The list of alternatives for HCFC-22 including high-GWP HFC replacements is given, and considerations regarding the choice of refrigerant, in both low-GWP synthetic and "natural" refrigerant alternatives, are presented. The low-GWP refrigerant flammability issue and the performance of equipment at high ambient temperature are described. It is discussed whether the potential impacts of the Kigali Amendment will be reinforcing the momentum of applications using low-GWP refrigerants and the innovation for sustainable RACHP technologies
\end{abstract}

Keywords: Montreal Protocol, HFCs, Kigali Amendment, HFC Regulations, Low-GWP Refrigerants.

\section{INTRODUCTION}

Chlorofluorocarbons (CFCs), hydrochlorofluorocarbons (HCFCs) and other ozone depleting substances (ODS) are potent greenhouse gases (GHG). The phase-out of these chemicals as mandated by the Montreal Protocol, and consequent resulting reductions of emissions and atmospheric concentrations have had an enormous contribution to climate protection, next to the original intent of the Montreal Protocol to protect the ozone layer. It has been estimated that the total avoided net annual ODS emissions would be equivalent to about $10 \mathrm{Gt} \mathrm{CO}_{2 \text {-eq }}$ in 2010, which is about five times the annual reduction target of the Kyoto Protocol for the period 2008-2012 [1].

According to studies and assessments [2, 3] the climate benefit of the Montreal Protocol could be reduced or totally lost in future if emissions of ODS substitutes with high global warming potentials (GWP), such as most HFCs, continue to increase. Based on these research results, parties to the Montreal Protocol started discussions (based on proposals) on an amendment to add HFCs and control schedules to the Montreal Protocol in 2009.

Hydrofluorocarbons (HFCs) were largely developed and promoted as alternatives to ODS and have been used in the last 30 years in several sectors, mainly as refrigerant in refrigeration, air conditioning and heat pumps (RACHP) applications. Most HFCs are greenhouse gases that can have high or very high-GWP, up to 14,800 [4].

The main issues that were thought to favor the inclusion of HFCs as controlled substances under the Montreal Protocol presented by the countries proposing amendments were: HFCs were developed and promoted as a result of Montreal Protocol CFC and HCFC control measures; the framework built by the Montreal Protocol for the phase-out of CFCs and HCFCs in the sectors where HFCs are being used, would be the most appropriate and effective method for the control of HFC production and consumption. On the other hand, the countries that initially were not in favor of such an amendment used arguments such as HFCs are no ODS and that would be the reason why they could not be included in an international agreement established for controlling ODS use. In the discussions that took place over the years, other issues were presented as barriers for including HFCs under the Montreal Protocol, such as financial support for the developing countries, the commercial availability of HFC alternatives, technology transfer and many others.

After seven years of intense discussions, the parties to the Montreal Protocol overcame the main obstacles for reaching a consensus decision, and at the 28th Meeting of the Parties on 15 October 2016 in Kigali, Rwanda, the parties decided on the 
addition of 17 HFCs to the Protocol (in a Group I). They are given with Global Warming Potentials using their IPCC AR4 report values [5], in a new Annex F. The annex also presents the GWP of CFCs and HCFCs. It also includes HFC-23 (in a Group II), a chemical which mainly originates as a by-product in HCFC-22 production facilities. Table 1 presents the information contained in the Annex F.

Table 1. Annex F to the Montreal Protocol [6]

\begin{tabular}{llll}
\multicolumn{2}{c}{ HFCs (Group I) } & \multicolumn{2}{c}{ HCFCs } \\
Substance & $\begin{array}{l}\text { GWP value } \\
(\mathbf{1 0 0} \text { year) }\end{array}$ & Substance & $\begin{array}{l}\text { GWP value } \\
(\mathbf{1 0 0} \text { year) }\end{array}$ \\
HFC-134 & 1100 & HCFC-21 & 151 \\
HFC-134a & 1430 & HCFC-22 & 1810 \\
HFC-143 & 353 & HCFC-123 & 77 \\
HFC-245fa & 1030 & HCFC-124 & 609 \\
HFC-365mfc & 794 & HCFC-141b & 725 \\
HFC-227ea & 3220 & HCFC-142b & 2310 \\
HFC-236cb & 1340 & HCFC-225ca & 122 \\
HFC-236ea & 1370 & HCFC-225cb & 595 \\
HFC-236fa & 9810 & & \\
HFC-245ca & 693 & & CFCs \\
HFC-43-10mee & 1640 & Substance & GWP value \\
HFC-32 & 675 & CFC-11 & 4750 \\
HFC-125 & 3500 & CFC-12 & 10900 \\
HFC-143a & 4470 & CFC-113 & 6130 \\
HFC-41 & 92 & CFC-114 & 10000 \\
HFC-152 & 53 & CFC-115 & 7370 \\
HFC-152a & 124 & & \\
\multicolumn{1}{c}{ HFCs (Group II) } & & \\
HFC-23 & 14800 & &
\end{tabular}

HFCs therefore became controlled substances under the Montreal Protocol, with specific HFC control schedules adopted for developing and developed countries (parties).

Developed (non-A5) countries will start to phase down HFCs by 2019. Developing countries (A5) will follow with a freeze of HFC consumption levels in 2024, with some countries freezing consumption in 2028.

The Kigali Amendment will enter into force on 1 January 2019, provided that it has been ratified by at least 20 Parties to the Montreal Protocol (or 90 days after ratification by the 20th Party, whichever is later).

\section{OVERVIEW OF THE KIGALI AMENDMENT}

The GWP values in the new Annex F must be used for the conversion of HFC mass quantities in in carbon dioxide equivalent $\left(\mathrm{CO}_{2} \mathrm{eq}\right)$ in all the reports countries need to present related to HFC phase-down implementation.

Including HFCs under the Montreal Protocol as controlled substances will not affect the obligations the countries have under the United Nations Framework Convention on Climate Change (UNFCCC). The amendment will not have the effect to exempt Parties of their commitments to send to UNFCC HFC emissions inventory reports (as established in Articles 4 and 12 of the UNFCCC). HFC consumption and production will be controlled under the Montreal Protocol while HFC emissions will continue to be reported under the UNFCCC.

The Kigali amendment has different years for HFC consumption used in the baseline and various phase-down schedules, i.e., two for two groups of Article 5 Parties (developing countries) and two for two groups of non-Article 5 Parties (developed countries). Figure 1 shows the baseline (freeze) and phase-down schedules.

The reason for including both HFCs and a percentage of HCFCs in the calculation of the baseline is due to the fact that
HFCs are thought to be utilized as alternatives for a certain portion of HCFCs still to be phased out. The HCFC component in the calculation is assumed to take this portion into account in the baseline.

In the reporting under the Montreal Protocol, the information about production, consumption, imports, exports and emissions of HFCs shall be expressed in $\mathrm{CO}_{2 \text {-eq }}$ and not in HFC mass quantities.
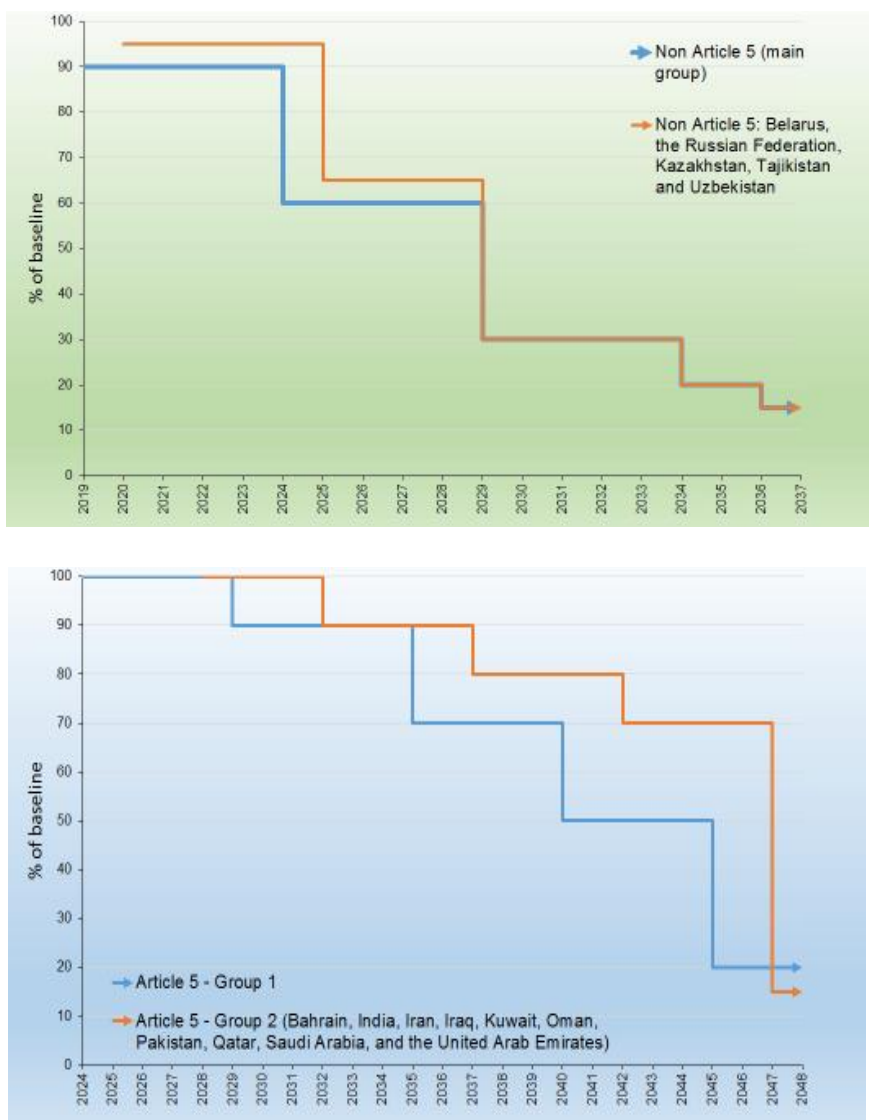

Figure 1. Kigali Amendment baseline calculation and phase-down schedules for non-A5 Parties (a) and A5 Parties (b) $[6]$

\subsection{Exemptions for High Ambient Temperature countries}

During the preparatory amendment discussions that took place during the last years, one of the issues that Parties had to deal with was the one presented by the countries experiencing high ambient temperatures (HAT). In these regions, due to high ambient temperature, the refrigerant condensing temperature in RACHP equipment is relatively high during part of the year and approaches the refrigerant critical temperature. For an air cooled system, the closer the critical temperature of the refrigerant is to the ambient temperature, the less efficient is the cycle with lower capacity, thus increasing energy consumption [7].

Operation of a RACHP system at high ambient temperatures intrinsically results in a lower coefficient of performance. This is the case for all refrigerants but the COP reduction is different among the various refrigerants. Over the years, countries experiencing HAT conditions expressed their concerns and worries of meeting an HFC freeze (date and level of consumption) as well as reduction targets. This is where low-GWP alternatives to HCFC-22 in small/medium size airconditioning applications are not yet introduced and verified 
by local markets. Some of these countries have already started to apply new minimum energy performance requirements.

In the discussion of the HFC amendment proposals, these issues were addressed. The solution agreed on was found in a different phase-down schedule for the countries experiencing high ambient temperatures, specifically India, Iran, Pakistan and the Gulf States. This exemption allows for a delay in the HFC freeze date and following phase-down obligations by a period of four years. It applies to the following equipment:

- Multi-split air conditioners (commercial and residential);

- Split ducted air conditioners (residential and commercial);

- Ducted commercial packaged (self-contained) air conditioners.

It is important to mention that considerations for equipment that will be operated at high ambient temperature conditions must not only be based on the choice of refrigerant but also on overall system design applied to obtain optimum and reliable performance under HAT conditions.

\section{POTENTIAL IMPACT TO REFRIGERANT CHOICE}

The Kigali amendment has reinforced the momentum towards applications using low-GWP refrigerants and accelerates innovation for sustainable RACHP technologies. One of the key issues for the Kigali amendment implementation is the replacement of HCFC-22 and highGWP HFCs with low-GWP refrigerants

Considering the R-410A and HCFC-22 replacement, the list of alternatives includes single-component or pure refrigerants, such as HFC-32, HC-290, HC-1270, R-717, R-744, and new refrigerant blends. These blends include the so-called hydrofluoroolefins (HFOs), unsaturated HFCs, such as HFO1234yf and HFO-1234ze(E), along with traditional (saturated) HFC refrigerants to achieve the desired properties of the blend, e.g., low-GWP, lower flammability, or lubricant compatibility [4]. Table 2 presents an overview of past, current and possible future refrigerant for the different RACHP applications.

Table 2. Overview of refrigerant use and alternatives to HCFCs and high-GWP HFCs [12, 13]

\begin{tabular}{|c|c|c|c|c|c|c|c|}
\hline Sector & CFCs & HCFCs & $\begin{array}{l}\text { HFCs } \\
\text { Pure \& } \\
\text { Blends }\end{array}$ & HCs & $\begin{array}{l}\text { CO2 } \\
\text { Ammonia }\end{array}$ & $\begin{array}{l}\text { Unsaturated } \\
\text { HFCs (HFOs) } \\
\text { Pure }\end{array}$ & $\begin{array}{l}\text { Blends with Unsaturated } \\
\text { HFCs (HFOs) }\end{array}$ \\
\hline $\begin{array}{l}\text { Domestic } \\
\text { Refrigeration }\end{array}$ & CFC-12 & & HFC-134a & HC-600a & Ammonia & HFC-1234yf & R-450A, R-513A,... \\
\hline \multirow{2}{*}{$\begin{array}{l}\text { Commercial } \\
\text { Refrigeration } \\
\text { (SA,CU,CS) }\end{array}$} & \multirow{2}{*}{$\begin{array}{l}\text { CFC-12 } \\
\text { R-502 }\end{array}$} & \multirow[t]{2}{*}{ HCFC-22 } & \multirow{2}{*}{$\begin{array}{l}\text { HFC-134a } \\
\text { R-404A } \\
\text { R-407A } \\
\text { R-407F }\end{array}$} & \multirow{2}{*}{$\begin{array}{l}\text { HC-600a } \\
\text { HC-290 }\end{array}$} & $\mathrm{CO2}$ & \multirow{2}{*}{$\begin{array}{l}\text { HFC-1234yf } \\
\text { HFC-1234ze(E) }\end{array}$} & \multirow{2}{*}{$\begin{array}{l}\text { R-450A, R-448A, R-444B, R-442A, } \\
\text { R-455A, R-450A, R-513A, R-448A, } \\
\text { R-449B,... }\end{array}$} \\
\hline & & & & & Ammonia & & \\
\hline \multirow[t]{2}{*}{$\begin{array}{l}\text { Transport } \\
\text { Refrigeration }\end{array}$} & & HCFC-22 & $\begin{array}{l}\text { HFC-134a } \\
\text { R-410A }\end{array}$ & \multirow[t]{2}{*}{$\begin{array}{l}\text { HC-290 } \\
\text { HC-1270 }\end{array}$} & \multirow[t]{2}{*}{$\mathrm{CO2}$} & \multirow[t]{2}{*}{ HFC-1234yf } & \multirow{2}{*}{$\begin{array}{l}\text { R-450A, R-448A, R-444B, } \\
\text { R-455A, R-446A, R-447A, R-447B, } \\
\text { R-448A, R-449A R-450A, R- } \\
513 A, \ldots\end{array}$} \\
\hline & & & R-407C & & & & \\
\hline $\begin{array}{l}\text { Industrial } \\
\text { refrigeration }\end{array}$ & & HCFC-22 & $\begin{array}{l}\text { HCFC-22 } \\
\text { HCFC-123 }\end{array}$ & $\begin{array}{l}\text { HC-1270 } \\
\text { HC-290 }\end{array}$ & $\begin{array}{l}\text { Ammonia } \\
\text { C02 }\end{array}$ & HFC-1234yf & $\begin{array}{l}\text { R-450A, “L-40”, R-444B, } \\
\text { R-455A, R-446A, R-447A, R- } \\
\text { 447B,R-450A, “XP-10”, R-448A, R- } \\
\text { 449A,... }\end{array}$ \\
\hline $\begin{array}{l}\text { Water heating } \\
\text { heat pumps }\end{array}$ & & HCFC-22 & $\begin{array}{l}\text { HCFO- } \\
\text { 1233zd(E) }\end{array}$ & $\begin{array}{l}\text { HC-290 HC- } \\
600 a\end{array}$ & $\begin{array}{l}\text { CO2 } \\
\text { Ammonia }\end{array}$ & $\begin{array}{l}\text { HFC-1234yf } \\
\text { HFC-1234ze(E) }\end{array}$ & $\begin{array}{l}\text { R-450A, “L-40”, R-444B, R-455A, } \\
\text { R-446A, R-447A, R-447B, R-450A, } \\
\text { R-513A, R-448A, R-449A,... }\end{array}$ \\
\hline $\begin{array}{l}\text { Air } \\
\text { Conditioners }\end{array}$ & CFC-12 & HCFC-22 & $\begin{array}{l}\text { HFC-134a HFC- } \\
32 \\
\text { R-410A } \\
\text { R-407C } \\
\end{array}$ & HC-290 & C02 & HFC-1234yf & $\begin{array}{l}\text { R-450A, “L-40”, R-444B, R-455A, } \\
\text { R-446A, R-447A, R-447B, R-450A, } \\
\text { R-513A, R-448A, R-449A,... }\end{array}$ \\
\hline \multirow[t]{2}{*}{ Chillers } & \multirow[t]{2}{*}{$\begin{array}{l}\text { CFC-12 } \\
\text { CFC-11 }\end{array}$} & $\begin{array}{l}\text { HCFC-22 } \\
\text { HCFC-123 }\end{array}$ & $\begin{array}{l}\text { HFC-134a } \\
\text { R-404A }\end{array}$ & \multirow[t]{2}{*}{$\begin{array}{l}\text { HC-290 HC- } \\
1270\end{array}$} & \multirow[t]{2}{*}{$\begin{array}{l}\text { Ammonia } \\
\text { C02 }\end{array}$} & $\begin{array}{l}\text { HFC-1234yf } \\
\text { HFC-1234ze(E) }\end{array}$ & \multirow{2}{*}{$\begin{array}{l}\text { R-450A, “L-40”, R-444B, R-455A, } \\
\text { R-446A, R-447A, R-447B, R-450A, } \\
\text { R-513A, R-448A, R-449A,... }\end{array}$} \\
\hline & & \begin{tabular}{|l} 
HCFO- \\
1233zd(E) \\
\end{tabular} & $\begin{array}{l}\text { R-410A } \\
\text { R-407C }\end{array}$ & & & $\begin{array}{l}\text { HFO- } \\
1336 \mathrm{mzz}(\mathrm{Z})\end{array}$ & \\
\hline $\begin{array}{l}\text { Mobile Air } \\
\text { Conditioner }\end{array}$ & CFC-12 & & $\begin{array}{l}\text { HFC-134a } \\
\text { R-410A } \\
\text { R-407C }\end{array}$ & & $\mathrm{CO2}$ & HFC-1234yf & $R-450 A, R-513 A$ \\
\hline
\end{tabular}

\section{Historical use}

\section{Current use on a commercial-scale}

Potentially feasible or limited use, and for demonstration, trials, niche applications, etc

In the last 3 years about 80 fluids, most of them blends containing HFOs, have been proposed for testing or are being tested in industry programmes, are pending publication, or have been published in ISO 817 and ASHRAE 34 refrigerant standards since the 2014 RTOC Assessment Report. The majority of these fluids are new mixtures [4].

Considering the probability of the development of new molecules (pure refrigerants), it is important to mention that significant efforts have been done in the past to find new fluids. A recent study [8] started with a database of over 150 million chemicals, screening more than 56,000 small molecules and finding none of them ideal. It can be concluded from the study that the prospects of discovering new chemicals that would offer better performance than the fluids currently known are minimal.

Considering specific RACHP applications, the following aspects can be mentioned. HFC-32 is an alternative for use in a certain range of middle size air conditioners, and there is an opportunity for a much wider application of hydrocarbons as well as in larger capacity commercial refrigeration equipment. 
The issue of hydrocarbon flammability (defined as "Class A3" refrigerant) is very important and it will need to be addressed via a revision of standards. This now is an ongoing discussion inside the international standards technical committees. Once this flammability issue will have been adequately addressed in standards, it may lead to the acceptance of larger quantities in equipment than possible at present. There is a recent European Commission report [9] on barriers posed by codes, standards and legislation to using climate-friendly technologies in the refrigeration, air conditioning, heat pumps and foam sectors.

In case of mobile air conditioning systems (MACs), a certain percentage portion may use R-744 (carbon dioxide), however, the majority is expected to use HFO-1234yf. For chillers, two pure HFOs, HFO-1234ze and HFO-1233zd, already commercialized, are now applied in larger chiller equipment.

Natural refrigerants such as R-744 are increasingly being used in supermarket systems worldwide - both in cascaded systems (R-744 for low temperature cascaded with a second refrigerant such as HFC-134a or similar and R-717 in limited cases) and in transcritical systems. Transcritical systems are being researched extensively to reduce their energy penalty at high ambient conditions through the use of component and system technologies such as ejector, adiabatic condensing, sub-cooling and parallel compression [4]. In lower ambient temperatures transcritical systems offer advantages associated with heat recovery and reuse in an adjacent heating/ hot water scheme. There are already some supermarket refrigeration systems installed in the field using these technologies.

The refrigerant selections that can be expected in the near future will be very much related to the perceived longer term "certainty" of low-GWP refrigerants, where the commercial availability, costs, energy efficiency, safety and servicing aspects will all be important. At present the choice is likely to be between the natural fluids (ammonia, $\mathrm{CO}_{2}$, hydrocarbons) in equipment developed for their use and more expensive synthetic fluids (HFO, HCFO, HFC/HFO blends) in the types of equipment as used for HCFCs and HFCs. Considering the $\mathrm{HFC} / \mathrm{HFO}$ blends, the question is whether they will be restricted to equipment where no major redesign is being planned, or can also be new, totally re-engineered designs. It is likely that there can and will only be a very limited amount of HFC-HFO blends in future $[10,11,12,13]$.

It is important to emphasize that the refrigeration, air conditioning and heat pump industry as well as the refrigerant servicing sector cannot be assumed to cope with the large number of HFC/HFO blends. Tables 3 and 4 present an overview of the present status of refrigerant alternatives published so far and proposed to HCFC and high-GWP replacements.

Table 3. Pure substances proposed to HCFCs and high-GWP HFCs replacement [6]

\begin{tabular}{|c|c|c|c|c|c|c|c|c|}
\hline $\begin{array}{l}\text { Refrigerant } \\
\text { Designation }\end{array}$ & $\begin{array}{l}\text { Proposed } \\
\text { to replace }\end{array}$ & $\begin{array}{l}\text { Safety } \\
\text { Class }\end{array}$ & $\begin{array}{l}\text { Chemical } \\
\text { Formula }\end{array}$ & $\begin{array}{l}\text { Molecular } \\
\text { Weight }\end{array}$ & $\begin{array}{l}\text { Boiling } \\
\text { Point }\left({ }^{\circ} \mathrm{C}\right)\end{array}$ & $\begin{array}{l}\text { ATEL/ } \\
\text { ODL } \\
\left(\mathrm{kg} / \mathrm{m}^{3}\right)\end{array}$ & $\begin{array}{l}\text { LFL } \\
\left(\mathrm{kg} / \mathrm{m}^{3}\right)\end{array}$ & GWP \\
\hline HFC-32 & $\begin{array}{l}\text { R-404A, } \\
R-410 A^{\times}\end{array}$ & A2L & $\mathrm{CH}_{2} \mathrm{~F}_{2}$ & 52,0 & -52 & 0,30 & 0,307 & 675 \\
\hline HC-290 & $\begin{array}{l}\text { HCFC-22, } \\
\text { R-404A, } \\
\text { R-407C }\end{array}$ & A3 & $\mathrm{CH}_{3} \mathrm{CH}_{2} \mathrm{CH}_{3}$ & 44,1 & -42 & 0,09 & 0,038 & \\
\hline HC-600a & HFC-134a & A3 & $\begin{array}{l}\mathrm{CH}\left(\mathrm{CH}_{3}\right)_{2-}- \\
\mathrm{CH}_{3}\end{array}$ & 58,1 & -12 & 0,059 & 0,043 & \\
\hline R-717 & $\begin{array}{l}\text { HCFC-22, } \\
\text { R-407C }\end{array}$ & B2L & $\mathrm{NH}_{3}$ & 17,0 & -33 & 0,00022 & 0,116 & \\
\hline R-744 & $\begin{array}{l}\text { R-404A, } \\
R-410 A\end{array}$ & $\mathrm{~A} 1$ & $\mathrm{CO}_{2}$ & 44,0 & $-78^{\circ}$ & 0,072 & $\mathrm{NF}$ & 1 \\
\hline $\begin{array}{l}\text { HCFO- } \\
\text { 1233zd(E) }\end{array}$ & HCFC-123 & A1 & $\begin{array}{l}\mathrm{CF}_{3} \mathrm{CH}= \\
\mathrm{CHCl}\end{array}$ & 130,5 & 18,1 & 0 & $\mathrm{NF}$ & 1 \\
\hline HF0-1234yf & HFC-134a & A2L & $\mathrm{CF}_{3} \mathrm{CF}=\mathrm{CH}_{2}$ & 114,0 & $-29,4$ & 0,47 & 0,289 & $<1$ \\
\hline $\begin{array}{l}\text { HFO- } \\
\text { 1234ze(E) }\end{array}$ & HFC-134a & A2L & $\begin{array}{l}\mathrm{CF}_{3} \mathrm{CH}= \\
\mathrm{CHF}\end{array}$ & 114,0 & $-19,0$ & 0,28 & 0,303 & $<1$ \\
\hline HC-1270 & $\begin{array}{l}\text { HCFC-22, } \\
\text { R-407C }\end{array}$ & A3 & $\begin{array}{l}\mathrm{CH}_{3} \mathrm{CH}= \\
\mathrm{CH}_{2}\end{array}$ & 42,1 & -48 & 0,0017 & 0,046 & \\
\hline $\begin{array}{l}\text { HFO- } \\
1336 \mathrm{mzz}(\mathrm{Z})\end{array}$ & HCFC-123 & A1 & $\begin{array}{l}\mathrm{CF}_{3} \mathrm{CH}=\mathrm{CH}- \\
\mathrm{CF}_{3}\end{array}$ & 164,1 & 33,4 & 0 & $\mathrm{NF}$ & 2 \\
\hline HCC-1130(E) & HCFC-123 & B2 & $\mathrm{CHCl}=\mathrm{CHCl}$ & 96,9 & 47,7 & & & $<1$ \\
\hline
\end{tabular}


Table 4. Blend refrigerants proposed to HCFCs and high-GWP HFCs replacement

\begin{tabular}{|c|c|c|c|c|c|c|}
\hline $\begin{array}{l}\text { Refrigerant } \\
\text { Designation }\end{array}$ & $\begin{array}{l}\text { Refrigerant } \\
\text { development } \\
\text { name }\end{array}$ & $\begin{array}{l}\text { Proposed } \\
\text { to replace }\end{array}$ & $\begin{array}{l}\text { Safety } \\
\text { Class }\end{array}$ & $\begin{array}{l}\text { Composition } \\
(\%)\end{array}$ & $\begin{array}{l}\text { Bubble point/ } \\
\text { dew or } \\
\text { Normal boiling } \\
\text { point }\left({ }^{\circ} \mathrm{C}\right)\end{array}$ & GWP \\
\hline & XP30 & HCFC-123 & B1 & $\begin{array}{l}\text { R-1336mzz(Z)/1130(E) } \\
(74,7 / 25,3)\end{array}$ & & 1,7 \\
\hline- & ARM-41a & HFC-134a & A1 & $\begin{array}{l}R-134 a / 1234 y f / 32 \\
(63 / 31 / 6)\end{array}$ & & 860 \\
\hline R-513A & XP10 & HFC-134a & A1 & $\mathrm{R}-1234 \mathrm{yf} / 134 \mathrm{a}(56 / 44)$ & $-29,2$ & 570 \\
\hline- & $\mathrm{N}-13 \mathrm{a}$ & HFC-134a & A1 & $\begin{array}{l}\text { R134a/1234ze(E)/1234yf } \\
(42 / 40 / 18)\end{array}$ & & 550 \\
\hline R-450A & $\mathrm{N}-13 \mathrm{~b}$ & HFC-134a & A1 & $\mathrm{R}-1234 \mathrm{ze}(\mathrm{E}) / 134 \mathrm{a}(58 / 42)$ & $-23,4 /-22,8$ & 550 \\
\hline R-515A & HDR-115 & HFC-134a & A1 & $\mathrm{R}-1234 \mathrm{ze}(\mathrm{E}) / 227$ ea $(88 / 12)$ & $-19,2$ & 400 \\
\hline R-513B & & HFC-134a & A1 & $\begin{array}{l}\text { R-1234yf/134a } \\
(58,5 / 41,5)\end{array}$ & $-29,9$ & 540 \\
\hline- & $\mathrm{D}-4 \mathrm{Y}$ & HFC-134a & A1 & $\begin{array}{l}\text { R-1234yf/134a } \\
(60 / 40)\end{array}$ & & 520 \\
\hline- & AC5X & HFC-134a & A1 & $\begin{array}{l}\text { R-1234ze(E)/134a/32 } \\
(53 / 40 / 7)\end{array}$ & & 570 \\
\hline- & ARM-42a & HFC-134a & A2L & $\begin{array}{l}R-1234 y f / 152 a / 134 a \\
(82 / 11 / 7)\end{array}$ & & 110 \\
\hline$R-444 A$ & AC5 & HFC-134a & A2L & $\begin{array}{l}\text { R-1234ze(E)/32/152a } \\
(83 / 12 / 5)\end{array}$ & $-34,3 /-24,3$ & 89 \\
\hline R-445A & AC6 & HFC-134a & A2L & $\begin{array}{l}\text { R-744/134a/1234ze(E) } \\
(6 / 9 / 85)\end{array}$ & $-50,3 /-23,5$ & 120 \\
\hline - & $\mathrm{R} 290 / \mathrm{R} 600 \mathrm{a}$ & $\mathrm{HFC}-134 \mathrm{a}$ & A3 & $\begin{array}{l}\text { R-600a/290 } \\
(60 / 40)\end{array}$ & & \\
\hline R-456A & & HFC-134a & $\mathrm{A} 1$ & $\begin{array}{l}\text { R-32/134a/1234ze(E) } \\
(6 / 45 / 49)\end{array}$ & $-31,1 /-25,7$ & 630 \\
\hline R-407G & & HFC-134a & A1 & $\begin{array}{l}\mathrm{R}-32 / 125 / 134 \mathrm{a} \\
(2,5 / 2,5 / 95,0)\end{array}$ & $-29,1 /-27,2$ & 1300 \\
\hline- & LTR4X & $\begin{array}{l}\text { HCFC-22, } \\
\text { R- } 407 \text { C }\end{array}$ & A1 & $\begin{array}{l}\text { R-1234ze(E)/32/125/134a } \\
(31 / 28 / 25 / 16)\end{array}$ & & 1200 \\
\hline R-514A & XP30 & HCFC-123 & B1 & $\begin{array}{l}\mathrm{R}-1336 \mathrm{mzz}(\mathrm{Z}) / 1130(\mathrm{E}) \\
(74,7 / 25,3)\end{array}$ & $29,0 / 29,0$ & 2 \\
\hline- & $\mathrm{N}-20$ & $\begin{array}{l}\text { HCFC-22, } \\
\text { R-407C }\end{array}$ & A1 & $\begin{array}{l}\text { R134a/1234ze(E)/1234yf/ } \\
32 / 125 \\
(31,5 / 30 / 13,5 / 12,5 / 12,5)\end{array}$ & & 890 \\
\hline - & $\mathrm{D} 52 \mathrm{Y}$ & $\begin{array}{l}\text { HCFC-22, } \\
\text { R-407C }\end{array}$ & A2L & $\begin{array}{l}\text { R-1234yf/125/32 } \\
(60 / 25 / 15)\end{array}$ & & 890 \\
\hline- & $\mathrm{L}-20$ & $\begin{array}{l}\text { HCFC- } 22, \\
\text { R- } 407 \text { C }\end{array}$ & A2L & $\begin{array}{l}\text { R-32/1234ze(E)/152a } \\
(45 / 35 / 20)\end{array}$ & & 330 \\
\hline - & LTR6A & $\begin{array}{l}\text { HCFC-22, } \\
\text { R-407C }\end{array}$ & A2L & $\begin{array}{l}\text { R-1234ze(E)/32/744 } \\
(63 / 30 / 7)\end{array}$ & & 200 \\
\hline R-444B & $\mathrm{L}-20 \mathrm{a}$ & $\begin{array}{l}\text { HCFC- } 22, \\
\text { R- } 407 C\end{array}$ & A2L & $\begin{array}{l}\text { R-32/1234ze(E)/152a } \\
(41,5 / 48,5 / 10)\end{array}$ & $-44,6 /-34,9$ & 300 \\
\hline - & ARM-32a & $\begin{array}{l}\text { HCFC-22, } \\
\text { R-404A, } \\
\text { R-407C }\end{array}$ & A1 & $\begin{array}{l}\text { R-125/32/134a/1234yf } \\
(30 / 25 / 25 / 20)\end{array}$ & & 1400 \\
\hline R-442A & & $\begin{array}{l}\text { HCFC-22, } \\
\text { R-404A, } \\
\text { R-407C }\end{array}$ & A1 & $\begin{array}{l}\text { R32/125/134a/152a/227ea } \\
(31,0 / 31,0 / 30,0 / 3,0 / 5,0)\end{array}$ & $\begin{array}{c}-46,5 / \\
-39,9\end{array}$ & 1800 \\
\hline R-449B & & $\begin{array}{l}\text { HCFC-22, } \\
\text { R-404A, } \\
\text { R-407C }\end{array}$ & A1 & $\begin{array}{l}\text { R-32/125/1234yf/134a } \\
(25,2 / 24,3 / 23,2 / 27,3)\end{array}$ & $-46,1 /-40,2$ & 1300 \\
\hline R-449C & DR-93 & $\begin{array}{l}\text { HCFC-22, } \\
\text { R-407C }\end{array}$ & A1 & $\begin{array}{l}\text { R-32/125/1234yf/134a } \\
(20 / 20 / 31 / 29)\end{array}$ & $-45,5 /-38,5$ & 1100 \\
\hline R-453A & RS-70 & $\begin{array}{l}\text { HCFC- } 22, \\
\text { R-407C }\end{array}$ & A1 & $\begin{array}{l}\text { R-32/125/134a/227ea/600/ } \\
601 \mathrm{a} \\
(20,0 / 20,0 / 53,8 / 5,0 / 0,6 / 0,6)\end{array}$ & $-42,2 /-35,0$ & 1600 \\
\hline R-407H & & $\begin{array}{l}\text { HCFC-22, } \\
\text { R-407C }\end{array}$ & A1 & $\begin{array}{l}\mathrm{R}-32 / 125 / 134 \mathrm{a} \\
(32,5 / 15,0 / 52,5)\end{array}$ & $-44,6 /-37,6$ & 1400 \\
\hline
\end{tabular}


Table 4. (continued) Blend refrigerants proposed to HCFCs and high-GWP HFCs replacement

\begin{tabular}{|c|c|c|c|c|c|c|}
\hline $\begin{array}{l}\text { Refrigerant } \\
\text { Designation }\end{array}$ & $\begin{array}{l}\text { Refrigerant } \\
\text { development } \\
\text { name }\end{array}$ & $\begin{array}{l}\text { Proposed to } \\
\text { replace }\end{array}$ & $\begin{array}{l}\text { Safety } \\
\text { Class }\end{array}$ & $\begin{array}{l}\text { Composition } \\
(\%)\end{array}$ & $\begin{array}{c}\text { Bubble point/ } \\
\text { dew or } \\
\text { Normal boiling } \\
\text { point }\left({ }^{\circ} \mathrm{C}\right)\end{array}$ & GWP \\
\hline$R-458 A$ & $\mathrm{TdX} 20$ & $\begin{array}{l}\text { HCFC-22, } \\
\text { R-404A } \\
\text { R-507A }\end{array}$ & A1 & $\begin{array}{l}\mathrm{R} 32 / 125 / 134 \mathrm{a} / 227 \mathrm{ea} / 236 \mathrm{fa} \\
(20,5 / 4,0 / 61,4 / 13,5 / 0,6)\end{array}$ & $\begin{array}{c}-39,8 / \\
-32,4\end{array}$ & 1600 \\
\hline$R-460 A$ & & $\begin{array}{l}\text { HCFC-22, } \\
\text { R-404A }\end{array}$ & A1 & $\begin{array}{l}\mathrm{R}-32 / 125 / 134 \mathrm{a} / 1234 \mathrm{ze}(\mathrm{E}) \\
(12,0 / 52,0 / 14,0 / 22,0)\end{array}$ & $-44,6 /-37,2$ & 2100 \\
\hline$R-460 B$ & LTR4X & $\begin{array}{l}\text { HCFC-22, } \\
\text { R-404A }\end{array}$ & A1 & $\begin{array}{l}\text { R-32/125/134a/1234ze(E) } \\
(28,0 / 25,0 / 20,0 / 27,0)\end{array}$ & $-45,2 /-37,1$ & 1300 \\
\hline R-449A & DR-33 (XP40) & $\mathrm{R}-404 \mathrm{~A}$ & A1 & $\begin{array}{l}\text { R-32/125/1234yf/134a } \\
(24,3 / 24,7 / 25,3 / 25,7)\end{array}$ & $-46,0 /-39,9$ & 1300 \\
\hline- & $\mathrm{N}-40 \mathrm{a}$ & $\mathrm{R}-404 \mathrm{~A}$ & A1 & $\begin{array}{l}\mathrm{R}-32 / 125 / 134 a / 1234 \mathrm{ze}(\mathrm{E}) / \\
1234 \mathrm{yf} \\
(25 / 25 / 21 / 20 / 9)\end{array}$ & & 1200 \\
\hline- & $\mathrm{N}-40 \mathrm{~b}$ & $\mathrm{R}-404 \mathrm{~A}$ & A1 & $\begin{array}{l}\text { R-1234yf/32/125/134a } \\
(30 / 25 / 25 / 20)\end{array}$ & & 1200 \\
\hline R-452A & DR-34 (XP44) & $\mathrm{R}-404 \mathrm{~A}$ & A1 & $\begin{array}{l}\text { R-1234yf/32/125 } \\
(30 / 11 / 59)\end{array}$ & $-47,0 /-43,2$ & 1900 \\
\hline$R-452 C$ & ARM-35 & $\mathrm{R}-404 \mathrm{~A}$ & A1 & $\begin{array}{l}\text { R-32/125/1234yf } \\
(12,5 / 61,0 / 26,5)\end{array}$ & $-47,8 /-44,4$ & 2000 \\
\hline$R-448 A$ & $\mathrm{~N}-40 \mathrm{c}$ & $\mathrm{R}-404 \mathrm{~A}$ & A1 & $\begin{array}{l}\text { R-32/125/1234yf/134a/ } \\
1234 z e(E) \\
(26,0 / 26,0 / 20,0 / 21,0 / 7,0)\end{array}$ & $-45,9 /-39,8$ & 1300 \\
\hline- & R32/R134a & $\mathrm{R}-404 \mathrm{~A}$ & A2L & $\mathrm{R}-32 / 134 \mathrm{a}(50 / 50)$ & & 990 \\
\hline- & ARM-31a & $\mathrm{R}-404 \mathrm{~A}$ & A2L & $\begin{array}{l}\text { R-1234yf/32/134a } \\
(51 / 28 / 21)\end{array}$ & & 460 \\
\hline- & $\mathrm{L}-40$ & $\mathrm{R}-404 \mathrm{~A}$ & $\mathrm{~A} 2 \mathrm{~L}$ & $\begin{array}{l}\mathrm{R}-32 / 1234 \mathrm{ze}(\mathrm{E}) / 1234 \mathrm{yf} / \\
152 \mathrm{a}(40 / 30 / 20 / 10)\end{array}$ & & 290 \\
\hline$R-454 A$ & DR-7॰ & $\mathrm{R}-404 \mathrm{~A}$ & A2L & R-1234yf/32 (65/35) & $-48,4 /-41,6$ & 240 \\
\hline $\mathrm{R}-454 \mathrm{C}$ & DR-3 & $\mathrm{R}-404 \mathrm{~A}$ & A2L & R-1234yf/32 (78,5/21,5) & $-45,8 /-38,0$ & 150 \\
\hline$R-454 A$ & D2Y-65 & $\mathrm{R}-404 \mathrm{~A}$ & A2L & R-1234yf/32 (65/35) & $-48,4 /-41,6$ & 240 \\
\hline $\mathrm{R}-457 \mathrm{~A}$ & ARM-20a & $\mathrm{R}-404 \mathrm{~A}$ & $\mathrm{~A} 2 \mathrm{~L}$ & $\begin{array}{l}\mathrm{R}-32 / 1234 y f / 152 a \\
(18 / 70 / 12)\end{array}$ & & 140 \\
\hline - & ARM-30a & $\mathrm{R}-404 \mathrm{~A}$ & $\mathrm{~A} 2 \mathrm{~L}$ & R-1234yf/32 (71/29) & & 200 \\
\hline$R-455 A$ & HDR-110 & $\mathrm{R}-404 \mathrm{~A}$ & A2L & $\begin{array}{l}\text { R-32/1234yf/744 } \\
(21,5 / 75,5 / 3)\end{array}$ & $-51,6 /-39,1$ & 150 \\
\hline - & $\mathrm{R} 32 / \mathrm{R} 134 \mathrm{a}$ & $\mathrm{R}-410 \mathrm{~A}$ & A2L & $\mathrm{R}-32 / 134 \mathrm{a}(95 / 5)$ & & 710 \\
\hline - & R32/R152a & $\mathrm{R}-410 \mathrm{~A}$ & A2L & $\mathrm{R}-32 / 152 \mathrm{a}(95 / 5)$ & & 650 \\
\hline- & DR-5 & $\mathrm{R}-410 \mathrm{~A}$ & $\mathrm{~A} 2 \mathrm{~L}$ & $\mathrm{R}-32 / 1234$ yf $(72,5 / 27,5)$ & & 490 \\
\hline- & $\mathrm{L}-41 \mathrm{a}$ & $\mathrm{R}-410 \mathrm{~A}$ & $\mathrm{~A} 2 \mathrm{~L}$ & $\begin{array}{l}\text { R-32/1234yf/1234ze(E) } \\
(73 / 15 / 12)\end{array}$ & & 490 \\
\hline- & $\mathrm{L}-41 \mathrm{~b}$ & $\mathrm{R}-410 \mathrm{~A}$ & A2L & R-32/1234ze(E) (73/27) & & 490 \\
\hline- & ARM-70a & $\mathrm{R}-410 \mathrm{~A}$ & $\mathrm{~A} 2 \mathrm{~L}$ & $\begin{array}{l}\mathrm{R}-32 / 1234 \mathrm{yf} / 134 \mathrm{a} \\
(50 / 40 / 10)\end{array}$ & & 470 \\
\hline- & HPR1D & $\mathrm{R}-410 \mathrm{~A}$ & A2L & $\begin{array}{l}\mathrm{R}-32 / 1234 \mathrm{ze}(\mathrm{E}) / 744 \\
(60 / 34 / 6)\end{array}$ & & 410 \\
\hline - & $\mathrm{D} 2 \mathrm{Y}-60$ & $\mathrm{R}-410 \mathrm{~A}$ & $\mathrm{~A} 2 \mathrm{~L}$ & R-1234yf/32 (60/40) & & 270 \\
\hline R-454B & DR-5A & $\mathrm{R}-410 \mathrm{~A}$ & A2L & $R-32 / 1234$ yf $(68,9 / 31,1)$ & $-50,9 /-50,0$ & 470 \\
\hline$R-452 B$ & $\begin{array}{l}\text { DR-55 } \\
(X L 55)\end{array}$ & $\mathrm{R}-410 \mathrm{~A}$ & A2L & $\mathrm{R}-32 / 1234 \mathrm{yf} / 125(67 / 26 / 7)$ & $-50,9 /-50,0$ & 680 \\
\hline$R-446 A$ & $\mathrm{~L}-41-1$ & $\mathrm{R}-410 \mathrm{~A}$ & A2L & $\begin{array}{l}\mathrm{R}-32 / 1234 \mathrm{ze}(\mathrm{E}) / 600 \\
(68,0 / 29,0 / 3,0)\end{array}$ & $-49,4 /-44,0$ & 460 \\
\hline R-447A & $L-41-2$ & $\mathrm{R}-410 \mathrm{~A}$ & $\mathrm{~A} 2 \mathrm{~L}$ & $\begin{array}{l}R-32 / 125 / 1234 z e(E) \\
(68,0 / 3,5 / 28,5)\end{array}$ & $-49,3 /-44,2$ & 570 \\
\hline$R-447 B$ & $\mathrm{~L}-41 \mathrm{z}$ & $\mathrm{R}-410 \mathrm{~A}$ & A2L & $\begin{array}{l}\text { R-32/125/1234ze(E) } \\
(68,0 / 8,0 / 24,0)\end{array}$ & $-50,3 /-46,2$ & 710 \\
\hline
\end{tabular}




\section{CONCLUDING REMARKS}

The adoption of the Kigali Amendment has reinforced the momentum towards applications using low-GWP refrigerants and is expected to accelerate innovation for sustainable RACHP technologies. Some HFC-free technologies face barriers to widespread uptake due to restrictive technical standards, in particular for flammable refrigerants. In order to enable transitions to flammable low-GWP refrigerants, a revision of the standard charge limits currently used is on the way.

The low GWP argument only cannot be expected to be the determining factor whether certain fluids will be considered. Energy efficiency, or rather, energy consumption reduction will be important. This is not only related to refrigerant thermo-physical properties, it is also determined by equipment design, system configuration, component efficiencies, operating conditions, system capacity, and system hardware.

The choice for refrigerants is very likely to be a combination of energy efficiency, costs, as well as environmental performance including safety aspects associated with refrigerant toxicity and flammability. Regional and national regulations (e.g. flammability and charge) will drive many developments that will take place.

The use of pure refrigerants, i.e., both HFOs and nonsynthetic "natural" refrigerants, including hydrocarbons, are expected to expand widely after 2019-2020, and this in a substantial amount of applications in various RACHP subsectors. It can already now be observed that there is a remarkably high level of activity in the RACHP equipment development sector, which is also reflecting to the commitment of companies engaged in this research and development to finding useful long term solutions in a market of ever-changing goals and objectives. As a result, the emphasis on equipment with improved energy efficiency (i.e., lower energy consumption levels) and refrigerants with a lowGWP is much more significant than before.

Both types of refrigerants, natural and synthetic, can and will co-exist in a near future, and can be complementary.

\section{REFERENCES}

[1] Velders G.J.M., Andersen S.O., Daniel J.D., Fahey D.W., McFarland M. (2007). The importance of the Montreal Protocol in protecting climate, PNAS 2007, Vol. 104, No. 12, pp. 4771-4772. DOI: $\underline{\text { 10.1073/iti.1207104 }}$

[2] Velders G.J.M., Ravishankara A.R., Miller M.K., Molina M., Alcamo J.J., Daniel J.S., Fahey D.W., Montzka S.A., Reiman S. (2012). Preserving Montreal Protocol climate benefits by limiting HFCs, Science, Vol. 335, pp. 922-923.

[3] Velders G.J.M., Solomon S., Daniel J.S. (2014). Growth of climate change commitments from HFC banks and emissions, Atmos. Chem. Phys., Vol. 14, pp. 45634572.

[4] UNEP. (2016). Decision XXVII/4 Task Force Report Further Information on Alternatives to Ozone Depleting Substances, UNEP, Ozone Secretariat, Nairobi, Kenya, ISBN 978-9960-076-17-5.

[5] IPCC. (2007). Contribution of Working Group I to the Fourth Assessment Report of the Intergovernmental Panel on Climate Change, Solomon S.D., Qin M. Manning Z., Chen M., Marquis K.B., Averyt M.,

Tignor and H.L. Miller (eds.), Cambridge University Press, Cambridge, United Kingdom and New York, NY, USA

[6] UNEP. (2017). The Kigali Amendment to the Montreal Protocol: HFC Phase-down. Retrieved online at: http://www.unep.fr/ozonaction/information/mmcfiles/ 7809-e-Factsheet_Kigali_Amendment to_MP.pdf

[7] Peixoto R., Polonara F., Pachai A., Nielsen O. (2016). Short course: challenges of natural working fluids in high ambient temperature countries, 12th IIR Gustav Lorentzen Natural Working Fluids Conference, Edinburgh, UK

[8] McLinden M.O., Kazakov A.F., Brown J.S., Domanski P.A. (2015). Hitting the bounds of chemistry: Limits and tradeoffs for low-GWP refrigerants, $24^{\text {th }}$ Int. Congress of Refrigeration, Yokohama, Japan, paper B1-We-1a.

[9] EC (2016). Report on the Barriers posed by codes, standards and legislation to using climate-friendly technologies in the refrigeration, air conditioning, heat pumps and foam sectors, retrieved online at: http://ec.europa.eu/transparency/regdoc/rep/1/2016/EN /COM-2016-749-F1-EN-MAIN-PART-1.PDF

[10] Aprea C., Greco A., Maiorino A., Masselli C., Metallo A. (2016). HFO1234yf as a drop-in replacement for R134a in domestic refrigerators: a life cycle climate performance analysis, International Journal of Heat and Technology, Vol. 34, Special Issue 2, pp. S212S218. DOI: $10.18280 /$ ijht.34Sp0204

[11] Kuijpers L.J.M. (2017). HFC phase-down schedules under the "Kigali" amended Montreal Protocol; potential impacts to the choice of alternatives, 7th Ammonia and CO2 Refrigeration Technologies, Ohrid, Republic of Macedonia, IIR.

[12] UNEP (2015). Report of the Refrigeration, Air Conditioning and Heat Pumps Technical Options Committee, 2014 Assessment, UNEP, Ozone Secretariat, Narirobi, Kenya, ISBN 078-9966-076-090 .

[13] UNEP (2015). Update Decision XXVI/9 Task Force report, Additional information on alternatives to ozonedepleting substances, UNEP, Ozone Secretariat, Narirobi, Kenya, ISBN 978-9966-76-14-4.

\section{NOMENCLATURE}

A5

AR

ASHRAE American Society of Heating, Refrigerating

ATEL

CFC

GHG

GWP

HAT

$\mathrm{HC}$

$\mathrm{HCFC}$

HFC

HFO

HPMP

IPCC

ISO

Countries operating under Article 5 of the Montreal Protocol (i.e. developing countries) Assessment report and Air-Conditioning Engineers Acute Toxicity Exposure Limit Chlorofluorocarbon

Greenhouse gas

Global Warming Potential

High ambient temperature

Hydrocarbons

Hydrochlorofluorocarbon

Hydrofluorocarbons

Hydrofluoroolefin (Unsaturated HFC)

HCFC Phase-out Management Plan International Panel on Climate Change International Organization for Standardization 
LFL

MAC

MLF

n- A5

ODL

ODP
Lower flammability level

Mobile air conditioning

Montreal Protocol Multilateral Fund

Countries not operating under Article 5 of the Montreal Protocol (i.e. developed countries)

Oxygen Deprivation Limit

Ozone Depleting Potential
ODS

RACHP

UNEP

UNFCCC

RTOC
Ozone Depleting Substances

Refrigeration, air Conditioning and heat pump

United Nations Environment Program

United Nations Framework Climate Change

\section{Convetion}

Refrigeration, Air Conditioning, Heat Pump

Technical Options Committee 